Pacific Journal of Mathematic

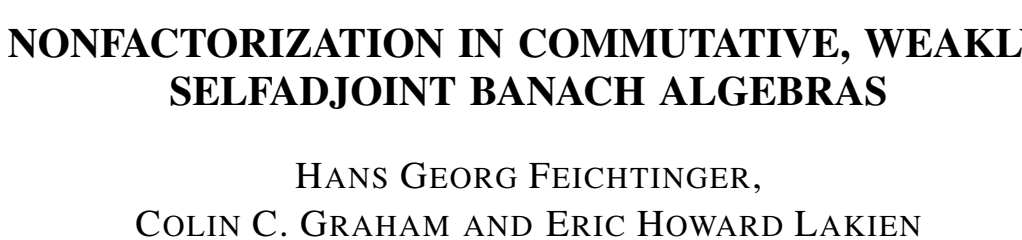




\title{
NONFACTORIZATION IN COMMUTATIVE, WEAKLY SELF-ADJOINT BANACH ALGEBRAS
}

\author{
Hans G. Feichtinger, Colin C. Graham and \\ ERIC H. LAKIEN
}

\begin{abstract}
A Banach algebra $A$ is said to have "(weak) factorization" if for each $f \in A$, there exist $g, h \in A$ (resp. $n \geqq 1$ and $\left.g,{ }_{1} h_{1}, \cdots, g_{n}, h_{n} \in A\right)$ such that $f=g h\left(f=\Sigma g_{j} h_{j}\right)$. Cohen's factorization theorem says that if $A$ has bounded approximate identity, then $A$ has factorization. The converse is false in general. This paper investigates various implications of factorization and weak factorization for commutative algebras that are weakly self-adjoint. (Defined below; these algebras include self-adjoint algebras.) The main result is Theorem 1.3: If the weakly self-adjoint commutative Banach algebra $A$ of functions on the locally compact space $X$ has weak factorization, then there exists $K>0$ such that, for all compact subsets $E$ of $X$, there exists $f \in A$ such that $\|f\| \leqq K$ and $f \geqq 1$ on $E$. Applications of 1.3 are given. In particular it is shown that a proper character Segal algebra on $L^{1}(G),(G$ a LCA group) cannot have weak factorization.
\end{abstract}

We say that a Banach algebra $B$ of complex-valued continuous functions on a topological space is weakly self-adjoint if there exists $K_{0}>0$ such that for each $f \in B$

$$
|f|^{2} \in B \text { and }\left\||f|^{2}\right\|_{B} \leqq K_{0}\|f\|_{B}^{2} \text {. }
$$

Obviously (0.1) is satisfied if $B$ is self-adjoint $(f \in B$ implies $\bar{f} \in B)$, or if $B$ is a Banach ideal of a self-adjoint Banach algebra $A$ of complex-valued continuous functions. Recall that $B$ is called a Banach ideal of a Banach algebra $A$ if $\|f\|_{B} \geqq\|f\|_{A}$ and $f g \in B$, with $\|f g\|_{B} \leqq$ $\|f\|_{B}\|g\|_{A}$ for all $f \in B, g \in A$. Dense Banach ideals have been called $A$-Segal algebras by Burnham [1] and others. It is worth mentioning that a self-adjoint Banach algebra $B$ has weak factorization if and only if each element of $B$ can be written as a linear combination of elements of the form $|f|^{2}, f \in B$ (this follows easily from the identity $\left.f \bar{g}=1 / 4 \sum_{k=0}^{3} f^{k}\left|f+i^{k} g\right|^{2}\right)$.

Our work was motivated by an attempt to give a converse to Cohen's theorem for Segal algebras on abelian groups (defined in $\S 2$ ), and thereby extend the results of Burnham [1], Leinert [7], Wang [15], Yap [17] and others. Since such a Segal algebra cannot have bounded approximate units, that would prove that a proper Segal algebra cannot have weak factorization. We cannot prove that in full generality, but we are able to improve earlier results substantially. 
Moreover, our results practically apply to all existing examples of Segal algebras. Special cases of some of the results of $\$ 2$ appeared already in [4].

1. Weak factorization. In this section we shall only deal with weakly self-adjoint Banach algebras of complex-valued continuous functions on a locally compact space $X$. To avoid trivialities we assume throughout that $B$ does not have an identity.

It is our aim to derive in this section certain properties for such a Banach algebra, if it has weak factorization. Since (weak) factorization is preserved under homomorphisms (in particular under the Fourier or Gelfand transform) these results can be used to prove nonfactorization for a number of subalgebras of $L^{1}(G)$ of a locally compact abelian group, in particular for most Segal algebras (cf. §2).

For later use let us denote the set $\{x \mid x \in X, f(x) \neq 0$ for some $f \in B\}$ by $S(B)$. We begin now with a lemma, abstracted from Lakien [4]:

LEMMA 1.1. If $B$ is a Banach algebra with weak factorization, then there exist positive integers $N$ and $K$ such that

$$
B(N, K):=\left\{f \in B: f=\sum_{i=1}^{N} \prod_{j=1}^{4} f_{i}^{j} \quad \text { with } \quad \sum_{i=1}^{N} \prod_{j=1}^{4}\left\|f_{i}^{j}\right\| \leqq K\|f\|\right\}
$$

is dense in $B$.

Proof. The assumption of weak factorization directly implies that $B=\bigcup_{N=1}^{\infty} \bigcup_{K=1}^{\infty} B(N, K)$. By the Baire category theorem, the closure of some $B\left(N^{\prime}, K^{\prime}\right)$ has nonempty interior. Thus, there exists some $f_{0} \in B\left(N^{\prime}, K^{\prime}\right)$ and some $\delta>0$ such that $B\left(N^{\prime}, K^{\prime}\right)^{-}$contains the ball of radius $\delta$ centered at $f_{0}$.

Let $f \in B$ be any function such that $\delta / 2 \leqq\|f\|<\delta$. Then for each $\delta / 4 \geqq \varepsilon>0$ there exist $\left(f_{i}^{j}\right)_{i=1}^{N^{\prime}}, j=1, \cdots, 4$ such that

$$
\left\|f+f_{0}-\sum_{i=1}^{N^{\prime}} \prod_{j=1}^{4} f_{i}^{j}\right\|>\varepsilon
$$

and

$$
\sum_{i=1}^{N^{\prime}} \prod_{j=1}^{4}\left\|f_{i}^{j}\right\|<K^{\prime}\left(\left\|f-f_{0}\right\|+\varepsilon\right) \leqq K^{\prime}\left(\varepsilon_{1} \delta_{1}\left\|f_{0}\right\|\right) \leqq K^{\prime}\left(2+\left\|f_{0}\right\|\right) .
$$

Since

$$
f_{0}=\sum_{i=1+N^{\prime}}^{2 N^{\prime}} \prod_{j=1}^{4} f_{i}^{j}
$$

with 


$$
\sum_{i=1+N^{\prime}}^{2 N^{\prime}} \prod_{j=1}^{4}\left\|f_{i}^{j}\right\| \leqq K^{\prime}\left\|f_{0}\right\|
$$

we see that

$$
\left\|\sum_{i=1}^{2 N^{\prime}} \sum_{j=1}^{4} f_{i}^{j}-f\right\|<\varepsilon
$$

and

$$
\sum_{i=1}^{2 N^{\prime}} \prod_{j=1}^{4}\left\|f_{i}^{j}\right\| \leqq \cdots 2 K^{\prime}\left(1+\left\|f_{0}\right\|\right)
$$

Note that

$$
\left\|\sum_{i}^{2 N^{\prime}} \Pi f_{i}^{j}\right\| \geqq \delta / 2-\varepsilon \geqq \delta / 4
$$

We set $N=2 N^{\prime}$ and $K=\delta K^{\prime}\left(1+1\left\|f_{0}\right\|\right) \delta^{-1}$ : then $f \in B$ and $\delta / 2 \leqq$ $\|f\| \leqq \delta$ imply $f \in B(N, K)$. Since $t B(N, K)=B(N, K)$ for all $t>0$ we see that $B=B(N, K)^{-}$.

The next lemma provides the key inductive step required in the proof of Theorem 1.3.

LEMMA 1.2. Let $B$ be a weakly self-adjoint Banach algebra with weak factorization. Then there exists a positive constant $K_{1}$ such that for every compact set $M \subset S(B)$ and every $f_{1} \in B$ vanishing nowhere on $M$, there exists $f_{2} \in B$ such that $f_{2} \geqq 0$

$$
f_{2} \geqq\left|f_{1}\right|^{1 / 2} \text {, and }\left\|f_{2}\right\| \leqq K_{1}\left\|f_{1}\right\|^{1 / 2} \text {. }
$$

Proof. According to 1.1 we can choose $f_{1}^{\prime} \in B(N, K)$ such that $\left\|f_{1}-f_{1}^{\prime}\right\| \leqq 1 / 4 \inf _{x \in M}\left|f_{1}(x)\right|$ (so necessarily $\left\|f_{1}-f_{1}^{\prime}\right\| \leqq\left\|f_{1}\right\|$ ). Write $f_{1}^{\prime}=\sum_{i=1}^{N} \Pi_{j=1}^{4} f_{i}^{j}$ with $\sum_{i=1}^{N} \prod_{j=1}^{4}\left\|f_{i}^{j}\right\| \leqq K\left\|f_{1}^{\prime}\right\|$. Without loss of generality, we may assume that

$$
\left\|f_{i}^{1}\right\|=\cdots=\left\|f_{i}^{4}\right\| \leqq\left(K\left\|f_{1}^{\prime}\right\|\right)^{1 / 4} \leqq\left(2 K\left\|f_{1}\right\|\right)^{1 / 4} .
$$

Set

$$
f_{2}^{\prime}=1 / 4 \sum_{i=1}^{N} \sum_{j=1}^{4}\left|f_{i}^{j}\right|^{2},
$$

so $f_{2}^{\prime} \geqq 0$ on $X$. By the weak self-adjointness, we have $f_{2}^{\prime} \in B$ and $\left\|f_{2}^{\prime}\right\| \leqq(2 K)^{1 / 2} N K_{0}\left\|f_{1}\right\|^{1 / 2}$.

Recall that (i) the geometric mean of $n$ nonnegative numbers does not exceed their arithmetic mean and (ii) the square root of the sum of $n$ nonnegative numbers does not exceed the sum of their square roots. We conclude that 


$$
f_{2}^{\prime} \geqq \sum_{i=1}^{N} \prod_{j=1}^{4}\left|f_{i}^{j}\right|^{1 / 2} \geqq\left(\sum_{i=1}^{N} \prod_{j=1}^{4}\left|f_{i}^{j}\right|\right)^{1 / 2} \geqq\left|f_{1}^{\prime}\right|^{1 / 2}
$$

Now $\left|f_{1}(x)-f_{1}^{\prime}(x)\right| \leqq|| f_{1}-f_{1}^{\prime}|| \leqq 1 / 4\left|f_{1}(x)\right|$ for all $x \in M$, so $\left|f_{2}^{\prime}\right| \geqq$ $(3 / 4)^{1 / 2}\left|f_{1}\right|^{1 / 2}$ on $M$. Therefore $f_{2}=4 / 3 f_{2}^{\prime}$ satisfies the required conditions with $K_{1}=2 K^{1 / 2} N K_{0}$, and the proof of the lemma is complete.

We now state the basic result of this paper.

THEOREM 1.3. Let $B$ be a weakly self-adjoint Banach algebra of complex-valued continuous functions. Suppose that $B$ has weak factorization. Then there exists $K_{2}>0$ such that for each compact subset $M \cong S(B)$ there is an element $f \in B$ such that $f \geqq 1$ on $M, f \geqq 0$ on $S(B)$, and $\|f\| \leqq K_{2}$.

Proof. We shall apply 1.2 inductively. First note that the weak self-adjointness of $B$ and the compactness of $M$ imply the existence of $f_{1} \in B$ such that $f_{1} \geqq 1$ on $M$. By 1.2 , there exist $f_{2}, f_{3}, \cdots \in B$ such that for $j=1,2, \cdots\left|f_{j+1}(x)\right| \geqq\left|f_{j}(x)\right|^{1 / 2}$ for $x \in M$ and $\| f_{j+1}|| \leqq$ $K_{1}\left\|f_{j}\right\|^{1{ }^{12}}$. Hence, $f_{j+1} \geqq 1$ on $M$ and $\left\|f_{j+1}\right\| \leqq K_{1}^{\sum_{i=0}^{j} 2^{-i}}\left\|f_{1}\right\| 2^{-j}$. Choose now $K_{2}=2 K_{1}^{2}$ and $j$ so large that $\left\|f_{1}\right\|^{2-j} \leqq 2$. Then $f=f_{j+1}$ will do, and the theorem is proved.

COROLLARY 1.4. Let $B$ be a weakly self-adjoint Banach algebra. Let $\mu$ be an unbounded, regular Borel measure on $S(B)$. If $B$ has weak factorization, then $B \nsubseteq \bigcup_{0<p<\infty} L^{p}(\mu)$.

This corollary is an immediate consequence of the above theorem, together with part (ii) of the following lemma.

Lemma 1.5. Let $\Omega$ be a space, $\Sigma$ a $\sigma$-algebra of subsets of $\Omega$, and $\mu$ a measure defined on $\Sigma$. We write $L^{p}$ in place of $L^{p}(\Omega, \Sigma, \mu)$.

(i) Let $B \cong L^{1}$ be a Banach space, continuously embedded in $L^{1}$. If $B \subseteq \bigcup_{1<p \leqq \infty} L^{p}$, then $B \subseteq L^{p_{0}}$ for some $p_{0}>1$.

(ii) Let $B \subseteq L^{\infty}$ be a Banach space, continuously embedded in $L^{\infty}$. If $B \cong \bigcup_{0<p<\infty} L^{p}$, then $B \subseteq L^{p_{0}}$ for some $p_{0}<\infty$.

Proof. (i) Since $B \cong L^{1}$, the hypothesis implies $B \subseteq \bigcup_{n=1}^{\infty} L^{1+1 / n}=$ $\bigcup_{k, n=1}^{\infty} k\left(B, L^{1+1 / n}\right)$, with $\left(B, L^{p}\right)=\left\{f \mid f \in B,\|f\|_{p} \leqq 1\right\}$. Since, by the reflexivity of $L^{p}, 1<p<\infty,\left(B, L^{p}\right)$ is closed in $B$, one of the sets $\left(B, L^{p}\right), 1<p<\infty$, has to have interior in $B$. Consequently $\left(B, L^{p}\right)-$ $\left(B, L^{p}\right) \cong 2\left(B, L^{p}\right)$ contains a neighborhood of the identity of $B$, and, hence, $B=L^{p}$. The proof of (ii) is similar.

Another consequence of Theorem 1.3 is that no proper Banach ideal of $C_{0}(X)$ can have factorization; but more is true. 
CoRollary 1.6. Let $B$ be a weakly self-adjoint Banach algebra with $X=S(B)$. Suppose that there exists a proper Banach ideal $N$ of $C_{c}(X)$ such that $N \supseteqq B$ and $S(N)=X$. Then $B$ does not have weak factorization.

Proof. We argue by contradiction, and suppose that $B$ has weak factorization. Then, by 1.3 and the closed graph theorem (applied to the inclusion of $B$ into $N$ ), there is $L>0$ such that, for each compact subset $M$ of $X$, there exists $f_{M} \in B$ such that $f_{M} \geqq 1$ on $M$ and $\left\|f_{M}\right\|_{N} \leqq L$. Let $g$ be such that $g=f_{M}^{-1}$ on $M$ and $\|g\|_{\infty} \leqq 1$. Then $\|h\|_{\infty} \leqq\|h\|_{N} \leqq\|g\|_{\infty}\|h\|_{\infty}\left\|f_{M}\right\|_{N} \leqq L\|h\|_{\infty}$ for all $h \in C_{0}(X)$ with compact support contained in $M$. This is not possible when $M$ is a proper Banach ideal of $C_{0}(X)$, vanishing nowhere on $X$.

2. Applications to Segal (and other) algebras, Let $G$ be a non-discrete locally compact abelian group. A Segal algebra on $G$ is a dense subspace of $L^{1}(G)$ that is a Banach space with a norm \| \| such that $\|f\| \geqq\|f\|_{1}$ for all $f \in B$ and such that for all $y \in G, f \in B$, $L_{y} f \in B\left(L_{y} f(x)=f\left(y^{-1} x\right)\right),\left\|L_{y} f\right\|=\|f\|$ and $\lim _{y \rightarrow 1}\left\|L_{y} f-f\right\|=0$ ([12], $\S 4$, or [11]). We say that a Segal algebra $B$ is character invariant if $\chi \in \widehat{G}, f \in B$ imply $\chi f \in B$. The most important property of a Segal algebra is the fact that it is a Banach ideal in $L^{1}(G)$. It is thus obvious that any Segal algebra on a locally compact abelian group can be considered as a weakly self-adjoint Banach algebra on $X=S(B)=\widehat{G}$ (via Fourier transform).

The main result of this section is Theorem 2.2. Proposition 2.1 is almost immediate from 1.4 and 1.6 and is stated here because it improves the main results of Wang [15] as well as of Burnham [1], Leinert [7], and Yap [17].

Proposition 3.1. Let $B$ be a Segal algebra on $G$. Then $B$ does not have weak factorization if one of the following conditions is satisfied:

(a) $B \cong \bigcup_{1<p \leqq \infty} L^{p}(G) ; \quad$ (b) $\hat{B} \cong \bigcup_{0<p<\infty} L^{p}(\hat{G})$;

(c) sup $\hat{f}(\gamma) M(\gamma)<\infty$ for all $f \in B$, with $M$ being an unbounded, continuous strictly positive function on $\hat{G}$.

Proof. (b) and (c) follow from $\$ 1$. If condition (a) is satisfied we use 1.5(i). Let $p_{0}$ be the number given in 1.5(1). Then if $1<p_{0}<2$, the Hausdorff-Young theorem implies $\hat{B} \subseteq L^{2}(G)$ and if $p_{0} \geqq 2$, the Plancherel theorem implies $\hat{B} \subseteq L^{2}(G)$, and (b) applies.

THEOREM 2.2. Let $B$ be a character invariant Segal algebra on 
the locally compact abelian group $G$. Then B has weak factorization if and only if $B=L^{1}(G)$.

REMARKs. Wang [15] and Leinert [7] have proved this result under additional hypotheses. For example, among others Wang needs $\|\chi f\|_{B}=\|f\|_{B}$ for all $\chi \in \hat{G}, f \in B$. (In this case, $B$ is called a character Segal algebra.) For character Segal algebras on compact abelian groups the result has been proved by Lakien [4]. It is his method that we extend below.

To prove 2.2 , we introduce a new algebra, $\widetilde{B}$, defined as follows. $\widetilde{B}$ consists of those measures $\mu \in M(G)$ such that there exists a net $\left\{f_{\alpha}\right\} \subseteq B$ with
(a) $\sup _{\alpha}\left\|f_{\alpha}\right\|_{B}<\infty$
and
(b) (weak-*) $\lim f_{\alpha}=\mu$.

The norm $\|\mu\|_{\widetilde{B}}$ is the infimum of the numbers (a) subject to (b). Obviously, $B$ is an ideal in $\widetilde{B}$, and $\widetilde{B}$ is an ideal $M(G)$, with $\|\mu * \nu\| \widetilde{B} \leqq$ $\|\mu\|_{M(G)}\|\nu\|_{\widetilde{B}}$, for all $\mu \in M(G), \nu \in \widetilde{B}$. If $G$ is compact, $B$ is closed in $\widetilde{B}$. (This is easily proved.) If $G$ is not compact, $B$ may not be closed in $\widetilde{B}$. (See [3], $\S 4$, Remark E) for an example.) The following lemma is Lemma 3.8 of [3].

LEMMA 2.3. Let $B$ be a character invariant Segal algebra. Then $B$ is closed in $\widetilde{B}$.

Lemma 2.4. Let $G$ be a locally compact group and $B$ a Segal algebra on $G$. If $B$ is closed in $\widetilde{B}$ and $\delta_{0} \in \widetilde{B}$, then $B=L^{1}(G)$.

Proof. Let $C>0$ be such that $C\|f\| \widetilde{B} \geqq\|f\|_{B}$ for all $f \in B$. Choose now now $\left\{g_{\alpha}\right\} \cong B$ such that (weak-*) $\lim g_{\alpha}=\delta_{0}$ and $\sup _{\alpha}\left\|g_{\alpha}\right\|_{B} \leqq$ $2\left\|\delta_{0}\right\|_{\widetilde{B}}$. Then $f=\left(\right.$ weak-*) $\lim g_{\alpha} * f, f \in B$. This implies $\|f\|_{\widetilde{B}} \leqq$ $\sup _{\alpha}\left\|g_{\alpha} * f\right\|_{B} \leqq 2\left\|\delta_{0}\right\| \widetilde{B}\|f\|_{1}$. Consequently, $B$ - and $L^{1}$-norms are equivalent on $B$ and $B=L^{1}(G)$.

Proof of 2.2. One direction is obvious. We prove the other. Suppose then that $B$ has weak factorization. Then by 1.3 there is a bounded net $\left\{f_{\alpha}\right\} \cong B$ such that for any compact subset $K \subseteq \hat{G}$ there is some $\alpha_{0}$ with $f_{\alpha}(t) \geqq 1$ for all $t \in K$ and $\alpha \geqq \alpha_{0}$. Without loss of generality, we may suppose that there is a bounded measure $\mu$ with $\mu=\left(\right.$ weak-*) $\lim f_{\alpha}$, i.e., $\mu \in \widetilde{B}$. We claim that $\hat{\mu}(t) \geqq 1$ for all $t \in \hat{G}$.

To see this, take $t_{0} \in \hat{G}$ and consider any continuous, nonnegative, compactly supported function $k$ on $\hat{G}$ with $\|k\|_{1}=1$. For sufficiently large $\alpha$ we have

$$
\widehat{f}_{\alpha}\left(t_{0} s^{-1}\right) \geqq 1
$$


for every $s$ in the support of $k$ and hence $k * \hat{f}_{\alpha}\left(t_{0}\right) \geqq 1$. Since $\breve{k} \in$ $C_{0}(G)$, the weak-* convergence of $\left\{f_{\alpha}\right\}$ to $\mu$ gives

$$
k * \widehat{f}_{\alpha}\left(t_{0}\right)=\left(\check{k} f_{\alpha}\right)^{\wedge}\left(t_{0}\right) \longrightarrow(\check{k} \mu)^{\wedge}\left(t_{0}\right)=k * \widehat{\mu}\left(t_{0}\right),
$$

so $k * \hat{\mu}\left(t_{0}\right) \geqq 1$ for every such $k$. Hence $\hat{\mu}\left(t_{0}\right) \geqq 1$, as required.

It is a well-known result of Wiener (see, e.g., Rudin [14], 5.6.9) that $\mu$ must have a nonzero discrete part. We may assume (using the translation-invariance of $\widetilde{B}$ ) that $\mu(\{0\}) \neq 0$. Let $g \in L^{1}(\widehat{G})$ be such that $g$ has compact support, $\widehat{g}(0)=1$, and

$$
\int_{G \backslash \backslash 0\}}|\hat{g}| d|\mu|<|\mu(\{0\})| / 4 \text {. }
$$

The character invariance of $\widetilde{B}$ implies that $\hat{g} \mu \in \widetilde{B}$ (cf. [3, proof of 3.8]). But $\hat{g} \mu=\mu(\{0\}) \delta_{0}+v$, where $\|v\|<|\mu(\{0\})| / 4$. Therefore $\hat{g} \mu$ is invertible in $M(G)$. Since $\widetilde{B}$ is an ideal in $M(G)$, this implies $\delta_{0} \in \hat{B}$ and the conclusion follows from 2.4 and 2.3.

The above result can be extended in the following way.

Corollary 2.5. Let $B$ a Banach algebra which is dense and continuously embedded in $L^{1}(G)$ of a locally compact abelian group. Suppose there exists $K_{0}>0$ such that

$$
f^{*} * f \in B \text { and }\left\|f^{*} * f\right\| \leqq K_{0}\|f\|^{2} \text { for all } f \in B \text {. }
$$

If $B$ has weak factorization, then $L^{1}(G)$ is the only character invariant Segal algebra on $G$ containing $B$.

Proof. Suppose $B$ is contained in a character invariant Segal algebra $B_{1}$. It then follows by the closed graph theorem that $B$ is continuously embedded in $B_{1}$. Since we may identify $B$ via Fourier transform with a weakly self-adjoint Banach algebra $\hat{B}$ on $\hat{G}$ with $S(\widehat{B})=\hat{G}$ it follows from 1.3 and the proof of 2.2 that $\widetilde{B}_{1}$ contains $\delta_{0}$ and thus $B_{1}=L^{1}(G)$. This completes the proof.

We now turn to some other applications of our methods.

TheOREM 2.6. If $I=\left\{f \in L^{1}(R), \hat{f}(t)=0\right.$ for $\left.0 \leqq t \leqq 1\right\}$, then $I$ does not have weak factorization.

Proof. Suppose that $I$ has weak factorization. Then, by 1.3 , there exists $K>0$ such that, for each compact subset $M \cong \boldsymbol{R}$ that is disjoint from $[0,1]$, there exists $f \in L^{1}(\boldsymbol{R})$ with $\|f\|_{1} \leqq K, \hat{f}=0$ on $[0,1]$ and $\hat{f} \geqq 1$ on $M$. For $n>0$ let $\mu=\mu_{n}$ be the measure on $R$ that assigns mass $1 / k$ to the point $-k / n$ and mass $-1 / k$ to the 
point $k / n$ for $1 \leqq k \leqq n$. Then $\sup _{r \in \boldsymbol{R}}|\hat{\mu}(r)| \leqq K_{1}$, where $K_{1}$ is independent of the integer $n$. Let $f \in I$ be such that $\|f\|_{1}<K$ and $\hat{f} \geqq 1$ on $\{-k / n: 1 \leqq k \leqq n\}$. Then $\hat{f} \mu>0$ and, therefore, $\left\|\hat{f}_{\mu}\right\|_{M(T)} \geqq$ $\sup _{r \in \boldsymbol{R}}\left|(\hat{f} \mu)^{\wedge}(r)\right| \geqq \delta \log n$. Since $\sup _{\boldsymbol{R}}\left|(\hat{f} \mu)^{\wedge}\right| \leqq\|f\|_{1} \sup _{\boldsymbol{R}}|\hat{\mu}|$, we have a contradiction.

That completes the proof of the theorem.

We conclude this section with a result concerning closed ideals of $L^{1}(G)$ (or any other Segal algebra on a compact abelian group $G$ ). A subset $E \subseteq \hat{G}$ is called a small $M_{0}$-set if $\hat{\mu} \in C_{0}(\hat{G})$ whenever $\hat{\mu}$ vanishes outside $E$. It is clear that any Sidon set or $\Lambda(p)$-set for $1<p<\infty$ (see [8] for definitions) is of this type. Moreover it is trivial that any Rajchman set in the sense of Pigno, [10], is a small $M_{0}$-set. Thus for example $-P \cup E$ is a Rajchman set if $E$ is a Rider set in $\hat{G}$ and $\hat{G}$ is an ordered group with positive cone $P$.

THEOREM 2.7. Let $G$ be a compact abelian group and $E \leqq \hat{G}$ a small $M_{0}$-set. Then the closed ideal

$$
I_{E^{c}}=\{f \mid \hat{f}=0 \text { off of } E\}
$$

has weak factorization if and only if $E$ is finite.

Proof. If $I_{E^{c}}$ has weak factorization then there exists $K>0$ such that for any finite subset $F \subseteq E$ there is some $f_{F} \in I_{E^{c}}$ with $\hat{f}_{F} \geqq 1$ on $F$ and $\|f\|_{L^{1}} \leqq K$. Taking a weak-* limit, we obtain $\mu \in M(G)$ such that $\widehat{\mu}(t)=0$ on $\widehat{G} \backslash E$ and $\hat{\mu} \geqq 1$ on $E$. This contradicts our assumptions concerning $E$, except the case that $E$ is finite. Since $I_{E^{c}}$ has a unit if $E$ is finite, the proof of the theorem is complete.

In view of the above remarks Theorem 2.7 includes Theorem 2.5 of [15] as well as the following result.

Corollary 2.8. (cf. Theorem 8.16 of [16])

$$
H^{1}=\left\{f \in L^{1}(T) \mid \hat{f}(n)=0 \quad \text { for } \quad n<0\right\}
$$

does not have weak factorization.

Proof. That $\{0,1,2 \cdots\}$ is a small $M_{0}$-set follows from the conjunction of the F. and M. Riesz theorem and the Riemann-Lebesgue lemma.

The authors are indebted to the referee for the kind permission to include the elegant proof of Lemma 2.2, and for several other helpful comments. 


\section{REFERENCES}

1. J. T. Burnham, Nonfactorization in subsets of the measure algebra, Proc. Amer. Math. Soc., 35 (1972), 104-106.

2. P. J. Cohen, Factorization in group algebras, Duke Math. J., 26 (1959), 199-205.

3. H. C. Feichtinger, Multipliers from $L^{1}(G)$ to a homogeneous Banach space, J. Math. Anal. Appl., 61 (1977), 341-356.

4. E. H. Lakien, Nonfactorization in Segal algebras on compact abelian groups, $\mathrm{Ph}$. D. Thesis, Northwestern University, 1975.

5. M. Leinert, A commutative Banach algebra which factorizes but has no approximate units, Proc. Amer. Mach. Soc., 55 (1976), 345-346.

6. - A factorable Banach algebra with unequivalent regular representation norm ..., Proc. Amer. Math. Soc., 60 (1976), 161-162.

7. — Remarks on Segal algebras, Manus, Math., 16 (1975), 1-9.

8. J. M. Lopez and K. A. Ross, Sidon Sets, Lecture Notes in Pure and Applied Mathematics, Marcel Dekker, New York 1974.

9. W. L. Paschke, A factorable Banach algebra without approximate unit, Pacific J. Math., 46 (1973), 249-251.

10. L. Pigno, Transforms vanishing at infinity and sums of Rider sets, Comm. Math. Helv. - to appear.

11. H. Reiter, Classical Harmonic Analysis and Locally Compact Groups, Oxford Univ. Press, Oxford 1968.

12. — $L^{1}$-algebras and Segal Algebras, Lecture Notes in Math., No. 231, SpringerVerlag, Berlin 1971.

13. M. Riemersma, On some properties of normed ideals in $L^{1}(G)$, Indag. Math., 37 (1975), 275-272.

14. W. Rudin, Fourier Analysis on Groups, Interscience, New York 1962.

15. H. C. Wang, Nonfactorization in group algebras, Studia Math., 42 (1972), 231-241. 16. - Homogeneous Banach Algebras, Lecture Notes in Pure and Applied Mathematics, M. Dekker, 29 (1977).

17. L. Y. H. Yap, Ideals in subalgebras of the group algebras, Studia Math., 35 (1970), 165-175.

Received April 28, 1977 and in revised form December 28, 1977.

MatheMATISChES INSTITUT DER UNIVERSITÄT

A-1090 Wien, Strudlhofgasse 4, Austria

NORTHWESTERN UNIVERSITY

Evanston, IL 60201

AND

14 Carluke Street

Toronto, Ontario, Canada 



\section{PACIFIC JOURNAL OF MATHEMATICS}

\section{EDITORS}

RICHARD ARENS (Managing Editor)

University of California

Los Angeles, California 90024

C. W. CURTIS

University of Oregon

Eugene, OR 97403

C. C. MOORE

University of California

Berkeley, CA 94720
J. DugundjI

Department of Mathematics University of Southern Californı Los Angeles, California 90007

R. Finn and J. Milgram Stanford University Stanford, California 94305

ASSOCIATE EDITORS

E. F. BECKENBACH

B. H. NeUmanN

F. WOLF

K. YoSHIDA

\section{SUPPORTING INSTITUTIONS}

UNIVERSITY OF BRITISH COLUMBIA CALIFORNIA INSTITUTE OF TECHNOLOGY

UNIVERSITY OF CALIFORNIA

MONTANA STATE UNIVERSITY

UNIVERSITY OF NEVADA, RENO

NEW MEXICO STATE UNIVERSITY

OREGON STATE UNIVERSITY

UNIVERSITY OF OREGON
UNIVERSITY OF SOUTHERN CALIFORNIA

STANFORD UNIVERSITY

UNIVERSITY OF HAWAII

UNIVERSITY OF TOKYO

UNIVERSITY OF UTAH

WASHINGTON STATE UNIVERSITY

UNIVERSITY OF WASHINGTON 


\section{Pacific Journal of Mathematics}

\section{Vol. 80, No. $1 \quad$ September, 1979}

Jeroen Bruijning and Jun-iti Nagata, A characterization of covering dimension by

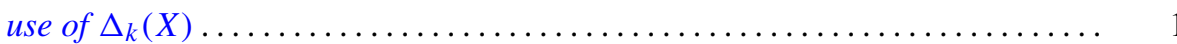

John J. Buoni and Albert Jonathan Klein, On the generalized Calkin algebra ...... 9

Thomas Ashland Chapman, Homotopy conditions which detect simple homotopy

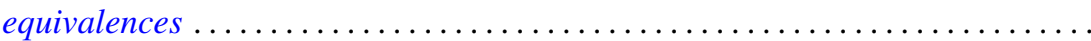

John Albert Chatfield, Solution for an integral equation with continuous interval

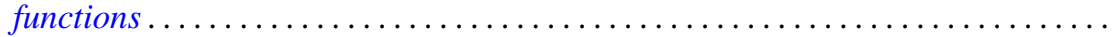

Ajit Kaur Chilana and Ajay Kumar, Spectral synthesis in Segal algebras on

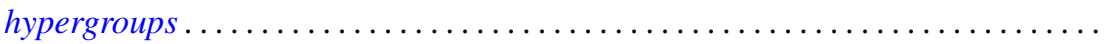

Lung O. Chung, Jiang Luh and Anthony N. Richoux, Derivations and

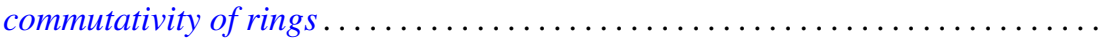

Michael George Cowling and Paul Rodway, Restrictions of certain function spaces to closed subgroups of locally compact groups .....................

David Dixon, The fundamental divisor of normal double points of surfaces........

Hans Georg Feichtinger, Colin C. Graham and Eric Howard Lakien,

Nonfactorization in commutative, weakly selfadjoint Banach algebras . . . . . . .

Michael Freedman, Cancelling 1-handles and some topological imbeddings ....... .

Frank E., III Gerth, The Iwasawa invariant $\mu$ for quadratic fields . . . . . . . . . . . . . .

Maurice Gilmore, Three-dimensional open books constructed from the identity

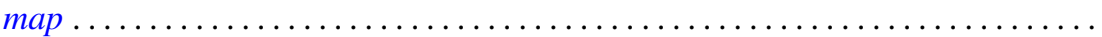

Stanley P. Gudder, A Radon-Nikodým theorem for $*$-algebras .

Peter Wamer Harley, III and George Frank McNulty, When is a point Borel? .

Charles Henry Heiberg, Fourier series with bounded convolution powers . .

Rebecca A. Herb, Characters of averaged discrete series on semisimple real Lie

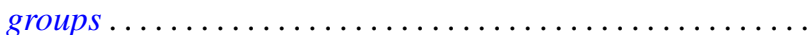

Hideo Imai, On singular indices of rotation free densities . .

Sushil Jajodia, On 2-dimensional CW-complexes with a single 2-cell . . .

Herbert Meyer Kamowitz, Compact operators of the form $u C_{\varphi}$

Matthew Liu and Billy E. Rhoades, Some properties of the Chebyshev method...

213

George Edgar Parker, Semigroups of continuous transformations and generating

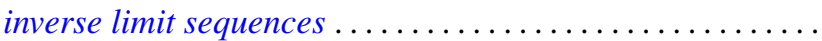

Samuel Murray Rankin, III, Oscillation results for a nonhomogeneous

equation ...

Martin Scharlemann, Transverse Whitehead triangulations ...

Gary Joseph Sherman, A lower bound for the number of conjugacy classes in a

finite nilpotent group

Richard Arthur Shoop, The Lebesgue constants for $\left(f, d_{n}\right)$-summability .

Stuart Jay Sidney, Functions which operate on the real part of a uniform

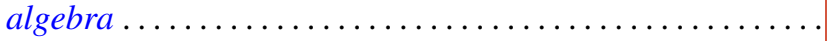

Tim Eden Traynor, The group-valued Lebesgue decomposition 